\title{
LA EDUCACIÓN Y ALFABETIZACIÓN DE JÓVENES Y ADULTOS EN REGIONES DEL CARIBE COLOMBIANO Y SU IMPACTO EN EL TRABAJO Y LOS INGRESOS*
}

\author{
Nydia Nina Valencia Jimenez ${ }^{1}$ \\ Jorge Eliecer Ortega Montes ${ }^{1}$ \\ Rubby Cecilia Castro Puche ${ }^{1}$
}

\begin{abstract}
RESUMEN: Se evalúan los impactos en el trabajo y los ingresos producidos por los jóvenes y adultos beneficiados del Programa de Educación y Alfabetización de Jóvenes y Adultos en Cartagena y el Departamento de Córdoba. El estudio se apoya en la teoría de las capacidades humanas y la estadística descriptiva. Como logros se destacan el incremento de las oportunidades al trabajo, la aplicación de nuevos conceptos, en contraposición se identificó como debilidades la poca ayuda brindada por el Estado y la insignificante variación de los ingresos. Se concluye que la educación por sí solo no garantiza el acceso a un trabajo digno ni una participación consciente en la transformación del contexto
\end{abstract}

Palabras claves: Educación de adultos. Economía de la educación. Evaluación política educativa. Colombia

\section{EDUCATION AND LITERACY OF YOUTH AND ADULTS IN COLOMBIAN CARIBBEAN REGION AND ITS IMPACT ON WORK AND INCOME}

ABSTRACT: Is evaluated the impact in work and income produced by beneficiaries of the Program Education and Literacy of young and adults in Cartagena and Cordoba Department. The study is supported on the theory of human capacities and the descriptive statistics. Some of the achievements are the increase of job opportunities, the application of new concepts, as opposed weaknesses identified himself as the little support and little variation of income. It's concludedthat education by

\footnotetext{
*Este artículo se fundamenta en uno de los resultados obtenidos del estudio de Impacto sociocultural y humano en regiones del caribe colombiano producido en desarrollo de las competencias de jóvenes y adultos del Programa de Alfabetización y Educación del Ministerio de Educación Nacional en el período 1997-2010 cofinanciado por la Universidad de Córdoba- Colombia y la Fundación para el Desarrollo Social Transformemos Educando, 2014.

${ }^{1}$ Universidad de Córdoba - Montería, Colombia. E-mails: nivaji@hotmail.com, paye51@hotmail.com, rubbycastro@yahoo.es

DOI: 10.1590/ES0101-73302017167856
} 
itself does not guarantee access to a decent job neither a conscious participation in the transformation of context.

Keywords: Adult education. Economics of education. Education policy evaluation. Colombia.

\section{L'ÉDUCATION ET L'ALPHABÉTISATION DES ADULTES ET JEUNES DANS LA RÉGION DES CARAÏBES COLOMBIEN ET SON IMPACT SUR LE TRAVAIL ET LE REVENU}

Résumé : On évalue l'impact dans le travail et le revenu des bénéficiaires du programme d'éducation et d'alphabétisation pour des jeunes et des adultes à Cartagena et dans le Département de Córdoba. L'étude est basée sur la théorie des capacités humaines et des statistiques descriptives. Comme réalisation on a eu l'augmentation de l'offre de travail et l'application de nouveaux concepts, alors que comme faiblesse on identifie la petite assistance fournie par l'État et le changement négligeable du revenu. On conclu que l'éducation elle-même ne garantit ni l'accès à un travail décent ni la participation consciente à la transformation de ce contexte.

Mots-clés : Léducation des adultes. L'économie de l'éducation. L'évaluation de la politique éducative. La Colombie.

\section{Introducción}

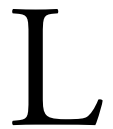

a constante preocupación de los organismos internacionales y del gobierno de Colombia en torno al problema de la Alfabetización y Educay educativa, que se manifiesta históricamente desde mediado de los años ochenta hasta la actualidad bajo distintos conceptos y modalidades de la política pública de educación, son hechos fehacientes que motivan esta investigación.

La Organización de las Naciones Unidas para la Educación, la Ciencia y la Cultura - UNESCO - (2015) señala que existen en el mundo alrededor de 781 millones de adultos analfabetos conservándose la tendencia entre el periodo 2000-2015 del 64\% de mujeres, desigualdad entre regiones y alzas significativas en el África Subsahariana (26\%) con pronósticos de incremento sobre el total de la población analfabeta de un 20 al 26\%. A luz de estas reflexiones el Informe de seguimiento de la educación en el mundo 2016 (UNESCO, 2016) hace un llamado contundente a intensificar las medidas para lograr el Objetivo 4 de Desarrollo Sostenible bajo el supuesto de que la educación es el eje transversal que potencia la 
consecución de los otros objetivos porque no solo permite la apropiación de competencias sino que coadyuva a la interpretación de la importancia del desarrollo sostenible en el futuro de la humanidad

En el caso de Colombia, según cifras del Plan Nacional de Desarrollo (2014-2018) se dice que en los últimos cuatro años se alfabetizaron cerca de 450.000 personas mayores de 15 años a lo largo de todo el territorio nacional. La tasa de deserción en educación básica y media se redujo del 4,9 \% en 2010 al 3,6 \% en 2013, y la brecha urbano-rural en cobertura neta de transición a educación media disminuyó siete puntos porcentuales, pasando de 15,5 en 2010 a 8,5 puntos en 2013 (DEPARTAMENTO NACIONAL DE PLANEACIÓN, 2014).

La Educación de Jóvenes y Adultos, como parte de la Política de educación nacional, surge y evoluciona en Colombia en el marco de las recomendaciones establecidas en las Conferencias de Jomtien (1990), Dakar (2000), la V CONFITEA (Conferencia Mundial de la Educación de Adultos) realizada en Alemania (1997) y la VI CONFITEA llevada a cabo en Belém do Pará (UNESCO, 2010)ํ․ Dicha política se asume como un instrumento clave para reducir las desigualdades y la pobreza, encaminada a promover destrezas y productividad en la población total en función de los cambios y necesidades de la economía y el mercado global, acogiendo igualmente las directrices que en tal sentido formuló la Comisión Económica para América Latina y el Caribe - CEPAL - y la Oficina Regional de Educación de la UNESCO para América Latina y el Caribe ORELAC (1992) para los países de la Región.

En la actualidad este tipo de educación es un componente estratégico del Sistema de Formación de Capital Humano SFCH, cuyos principios y propósitos se definen en los siguientes documentos de política económica y social: CONPES 2945 de 1997, CONPES Social 81 de 2004, CONPES 3527 de 2008 y CONPES 3582 de 2009; que a su vez orientan los siguientes planes prospectivos de desarrollo, Visión Colombia (2019), el Plan de Desarrollo Sectorial - La Revolución Educativa y el Plan Nacional de Desarrollo, 2010-2014; 2015-2018. Hoy día la ejecución de esta política educativa se promueve y se ofrece a través de programas especiales de educación flexibles a cargo del Ministerio de Educación Nacional MEN, quien ejerce las funciones de planeación, cofinanciación e inspección y vigilancia a nivel nacional, en tanto que su operación se delega a los entes territoriales quienes, en alianzas con distintos oferentes del sector solidario, desarrollan dichos programas mediante modelos pedagógicos diferentes con diversas formas de atención y de educación y alfabetización.

Es en este contexto donde se realiza la investigación que sustenta el presente artículo, que comprende solamente los resultados atinentes a uno de los objetivos propuestos, cual es el de evaluar los cambios ocurridos por esta población en los aspectos relacionados con el acceso al trabajo y la generación de ingresos monetarios. Para ello se parte del supuesto de que si los Programas de alfabeti- 
zación, educación básica y media de jóvenes y adultos que se impartieron en el Departamento de Córdoba y la ciudad de Cartagena -Colombia- en el período 1997-2010, consideran esta educación como un proceso formativo tendiente a que las personas desarrollen una capacidad de interpretar la realidad y de actuar, de manera transformadora en su contexto, haciendo uso creativo de los conocimientos, valores y habilidades a través de la lectura, escritura, matemática básica y la cultura propia, es factible esperar que las personas alfabetizadas como fruto de esos procesos, experimenten un cambio favorable en sus condiciones de vida, las oportunidades de trabajo y la generación de sus ingresos.

\section{Fundamentos teóricos y conceptuales}

El estudio realizado se apoya en la teoría de las capacidades humanas que nos permite asumir el concepto de competencias como la capacidad que dispone cada individuo en transformar sus recursos en libertades para el logro de sus propios fines, y el concepto de bienestar como el conjunto de cosas que una persona logra hacer o ser el vivir (SEN, 2000), o sea, como la capacidad que tiene una persona para ser, estar o hacer con sus bienes lo que mejor le parezca; en contraposición con el concepto hegemónico de bienestar que lo asocia estrictamente con el conjunto de bienes o cosas que se poseen y con la declaración formal del reconocimiento de libertades y derechos. Para este autor, por el contrario, el bienestar social también puede consistir en una mejoría en las condiciones materiales de vida de los individuos, en el aumento de la autoestima, la participación consciente y deliberada en los asuntos que les competen, en la posibilidad de elegir y ser elegidos, en tener oportunidades y opciones para el acceso y ejercicio del trabajo, la salud y la educación, entre otras. Con fundamento en esta teoría cuando aquí se habla de un cambio favorable esperado en las condiciones de vida, las oportunidades de trabajo y la generación de ingresos de la población objeto del estudio se está haciendo referencia es a la ampliación de las oportunidades, opciones y ayudas reales y prácticas de las personas que les permita incrementar sus capacidades humanas (CASAS; CORTÉS; GAMBOA, 2001).

En coherencia con el enfoque anterior, el concepto de alfabetización al que se hace referencia en nuestra investigación se aproxima al definido por la Conferencia Mundial de Educación para Todos, realizada en Jomtien (Tailandia, 1990), según el cual alfabetizar significa propiciar aprendizajes que permitan que las personas colectivamente puedan mejorar sus condiciones de vida, buscar alternativas para los problemas que afectan a sus comunidades y a sí mismas, y conocer nuevas formas de participar de la sociedad a la que pertenecen. Dicho concepto considera que las personas están permanentemente educándose en distintos ámbitos sociales, más allá de la escuela; en lugar de respuestas educativas uniformes y descontextualizadas. Bajo este concepto los 
programas educativos, incluyendo los de alfabetización de jóvenes y adultos, deberían estar conectados a las necesidades básicas de las poblaciones, y por eso su organización debería ser tan diversa como las singularidades de los contextos donde ocurren, en contraposición al concepto tradicional de carácter funcional que lo interpreta como un simple dominio del mecanismo de la escritura, con un único método para alfabetizar, textos construidos artificialmente y una única forma para el habla (KALMAN, 2002; TORRES, 2005).

\section{Metodología}

El universo la constituye la población de jóvenes y adultos mayores de 15 años del género femenino y masculino beneficiarias del Programa de alfabetización y educación que al menos hayan cursado el IV ciclo de los VI que componen la modalidad de este programa educativo ${ }^{2}$ que integran el plan de estudio de estos modelos en el departamento de Córdoba y la ciudad de Cartagena durante el período 1997-2010.

El tamaño de la muestra fue de 753 personas seleccionadas del listado de estudiantes matriculados en el Sistema de Matrículas del Ministerio de Educación Nacional y las bases de datos de la Fundación Social Transformemos Educando, uno de los oferentes y ejecutores de dichos modelos. Se utilizó un muestreo simple aleatorio con criterios geográficos y de género. Para el caso del Departamento de Córdoba se abarcó una porción representativa de la población universo residente en 18 municipios no certificados en educación.

La encuesta se estructuró en seis bloques de preguntas. El primer bloque recoge la información referente a los datos generales personales de cada uno de los encuestados. El Bloque dos (BII), se refiere a la información requerida para el logro del objetivo relacionado con los factores asociados al acceso, permanencia y continuidad en el sistema educativo. El bloque tres (BIII), a los cambios experimentados en el trabajo, los ingresos y el entorno sociocultural, el bloque cuatro (BIV) al desarrollo Humano y las competencias de los saberes. El cuestionario comprendió 20 preguntas en total con la modalidad de la escala Likert y valores de 1 a 5 , que permitieron someter a consideración de los encuestados un conjunto de afirmaciones como alternativas de respuesta debidamente ordenadas en función de sus respectivos valores. De modo que el valor 5 corresponde a la respuesta teóricamente esperada y el 1 a la menos evidente.

Para el análisis de los datos se realizó un muestreo estratificado no proporcional clasificando y definiendo como estratos tres grupos de estudiantes de la muestra, de acuerdo a la siguiente condición: estudiante ACTIVO, RETIRADO y EGRESADO. Ello permitió establecer tres tamaños de muestra según el detalle relacionado a continuación. 


\section{Identificación, definición y \\ selección de factores, categorías y/variables}

\section{Definición de estratos}

1. Estudiantes Activos, son aquellos estudiantes que al momento de la encuesta se encontraban cursando al menos el IV ciclo de los seis que componen la estructura curricular del Programa

2. Estudiantes Retirados, son los estudiantes que habiendo ingresado al Programa y cursado al menos el IV ciclo escolar no pudieron permanecer en el Programa, y

3. Estudiantes Graduados, son los estudiantes egresados del Programa que lograron culminar los seis ciclos escolares y alcanzaron el título de bachiller en cualquier año del periodo fijado en el estudio.

\section{Procedimiento}

\section{Identificación de factores, categorías y/o variables}

Los factores, las categorías y/o variables objeto de la evaluación se identificaron y definieron mediante la modalidad de preguntas múltiples con múltiples respuestas debidamente ordenadas según la valoración de la escala Likert, las cuales se asumieron como categorías y/o variables ordinales explicativas. Dichas categorías y/o variables explicativas, para el caso del objetivo relacionado con los cambios ocurridos en el acceso al trabajo y los ingresos, se seleccionaron de las respuestas dadas por los encuestados al Bloque III del cuestionario, que contiene las preguntas $\mathrm{p} 4, \mathrm{p} 5$, p6, p7, p8 y del Bloque IV que contiene las p9,p10,p11y p12. De esta manera se identificaron los ítem de mayor puntaje, o sea, las que marcan la tendencia positiva, las de puntaje medio y las de menor puntaje.

\section{Selección de las categorías y/o variables explicativas y del modelo de medición y validación}

Una vez identificadas las categorías de mayor puntaje, se tomaron los logaritmos naturales de los valores respectivos con el fin de estimar el antilogaritmo y poder realizar un análisis de varianza usando como criterio de clasificación los estratos pre-establecidos (graduado, retirado, activo). El antilogaritmo de la media de los logaritmos es la media geométrica, que permite comparar las medias geométricas. 


\section{Validez de la información}

Después de haber seleccionado las categorías y/variables explicativas se procedió a la validez de esa información mediante la comprobación de hipótesis, usando la prueba estadística de Rangos signados de Wilcoxon, (prueba no paramétrica), y formulando la Ho que la MEDIA de las respuestas de los encuestados a cada una de esas alternativas es mayor que 3 , es decir es 4 o 5 . Como no se rechaza esa hipótesis, no cabría duda que los encuestados consideran VERDADE$\mathrm{RO}$ esas afirmaciones. Se eligieron las medianas en lugar de las medias porque las respuestas son una variable categórica ordinal.

\section{Comparación de la información por estratos (Activos-graduados-retirados)-ANOVA}

Se hace una comparación de las MEDIAS de los tres estratos (activos, graduados, retirados), con una prueba estadística no paramétrica. De esa forma si no se puede rechazar la hipótesis de igualdad de las tres medianas, significa que la opinión es la misma, independientemente de que esté graduado o activo o retirado. También se calcularon las estadísticas básicas de cada categoría, en este caso se analizaron los cuartiles y la media, ya que las respuestas son categóricas ordinales (la media no tiene sentido). El primer cuartil igual a 2 (por ejemplo) significa que el $25 \%$ de los encuestados marcaron 2 o menos y el $75 \%$ marcaron por encima de 2 . Una media igual a 4 significa que el $50 \%$ de los encuestados marcaron 4 o más. 3. Posteriormente se hizo una prueba que involucra a las 5 categorías seleccionadas, que fueron las afirmaciones identificadas con los literales: a), b), c), d) y e) conjuntamente, obteniéndose un puntaje global mediante la suma de las calificaciones dadas por los encuestados, es decir, para cada encuestado se hace la suma $a)+b)+c)+d)+e$ ). La lógica nos dice que si todos marcaran 4 o 5 esa suma debería ser 20 o 25 , se demuestra mediante una prueba estadística $T$ usual que en promedio la suma es menor o igual que 15 (el valor de la suma si todos marcaran 3 o menos), como se rechaza esa hipótesis significa que en promedio la gente calificó 4 o 5, corroborando la Hipótesis Alternativa que los encuestados contestaron VERDADERO O COMPLETAMENTE VERDADERO A ESAS 5 CATEGORÍAS. Finalmente, usando ese puntaje global (suma) se comparan los tres grupos, para tratar de demostrar que independientemente de que estén activos, graduados o retirados, la opinión es la misma. Las comparaciones se hicieron mediante un Análisis de Varianza, ANOVA usual, (sin transformar), ya que se está comparando la suma y como tenemos un tamańo de muestra grande (más de 700) el teorema del límite central respalda ese procedimiento. 


\section{Resultados}

La mayoría de esta población es de género femenino y se encuentra en situación de pobreza y desplazamiento forzado, un alto porcentaje de ella lo representan mujeres cabeza de hogar, indígenas y afrocolombianos. Los ingresos para el sustento familiar se derivan del trabajo propio, oficios transitorios del sector público y privado, ventas callejeras, oficios varios y labores domésticas del hogar, los cuales complementan con subsidios provenientes del Programa presidencial "Familias en acción"3.

El mayor rango de edad está entre los 21 y 30 años, equivalente al 30\%, estimándose un $18 \%$ de la población con edades de hasta 20 años.

\section{Cambios ocurridos en las oportunidades de acceso al trabajo}

Se identificaron esas oportunidades y medios a través de los ítem (factores, categorías o variables explicativas) que los encuestados contestaron con puntajes más altos - verdadero o completamente verdadero - ( 4 o 5$)$ que son las afirmaciones: a) Mi oportunidad de acceso al trabajo se incrementó, b) La calidad de mi trabajo mejoró, c) Mi rendimiento laboral mejoró, d) Aplico nuevos conceptos y técnicas de trabajo que antes no conocía, y e) Hago un mejor aprovechamiento de mis recursos. Hay unas respuestas o categorías que el encuestado le parecen falso o completamente falso que haya ocurrido porque fueron calificados por la mayoría con 1 o 2, estos son: f) He recibido ayuda gubernamental para mi negocio, g) He recibido crédito bancario para mi negocio, h) Mis ventas se incrementaron, i) He conquistado nuevos clientes, j) Las condiciones de mi sitio de trabajo mejoraron

Los ítems de mayor puntaje fueron a, b, c, d, e, y los de menor puntaje fueron $\mathrm{f}, \mathrm{g}, \mathrm{h}, \mathrm{i}, \mathrm{j}$. A continuación se lleva a cabo la prueba de la hipótesis de que "la mediana es menor o igual a 3", frente a la hipótesis que «la mediana es mayor que 3" para cada uno de los ítems de mayor puntaje (a, b, c, d, e),

Un análisis comparativo entre los grupos o estratos adoptados (Activo- Graduado y Retirado) usando la prueba de Kruskal-Wallis de las Medianas para cada una de las categorías o variables en mención, permite establecer, para el caso de la categoría identificada con literal a) "Mi oportunidad de acceso al trabajo se incrementó", que la media de los activos es 3 y está por debajo de la de los graduados y retirados que es 4 (a eso se debe el rechazo de la Ho). Los activos son los que más Baja valoración le dieron a esa categoría, que en su gran mayoría calificaron con 3 (ni verdadero ni falso), los graduados y retirados calificaron en su gran mayoría 4, pero también hay un alto porcentaje que calificaron 3 o 2 . En síntesis se puede concluir que hay una variación más grande en la respuesta 
de retirados y graduados que en la respuesta de los activos, lo que quiere decir que hay un mayor consenso entre los activos en cuanto la valoración de esa respuesta como verdad. Sin embargo, es evidente una tendencia de la mediana a ser mayor que 3 .

Finalmente, una prueba que involucra a las 5 categorías de respuestas: a), b), c), d) y e) conjuntamente, permite obtener un puntaje global mediante la suma de las calificaciones dadas por los encuestados, es decir, para cada encuestado se hace la suma a), b), c), d) y e). La lógica nos dice que si todos marcaran 4 o 5 esa suma debería ser 20 o 25 . Luego para corroborar eso se hace la prueba estadística: Ho: «el promedio es menor o igual que 15» frente a H1: «El promedio es mayor que 15» (el valor 15 es el que tendría la suma si todos los encuestados calificaran $3(\mathrm{NI})$ ), Si se rechaza la hipótesis nula Ho, lo que significa es que en promedio la gente calificó 4 o 5 . De esta manera se corrobora la Hipótesis alternativa que afirma que los encuestados marcaron esas categorías como VERDADERAS.

Se comparan los tres grupos para tratar de demostrar que independientemente de que estén activos, graduados o retirados, la opinión es la misma.

En los datos de la Tabla 1 se puede observar que hay una diferencia significativa entre el promedio de los puntajes dados por los activos, graduados y retirados. No hay diferencia entre retirados y graduados en cuanto a su opinión mientras que hay diferencia en la opinión de los Retirados frente a los Activos y también de los

\section{Tabla 1}

Cambios en las oportunidades de acceso al trabajo según la opinión de la población encuestada por estratos.

\begin{tabular}{l|c|c|c|c}
\hline Estrato & diff & lwr & upr & p adj \\
\hline Activo-Graduado & -2.2322 & -4.1786 & -0.2858 & 0.0198 \\
\hline Retirado-Graduado & 0.2047 & -1.0536 & 1.4631 & 0.9227 \\
\hline Retirado-Activo & 2.4370 & 0.2851 & 4.5888 & 0.0218 \\
\hline
\end{tabular}

Analysis of Variance Table.

Response: y

Df Sum Sq Mean Sq F value $\operatorname{Pr}(>\mathrm{F})$

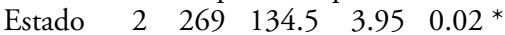

Residuals $71424332 \quad 34.1$

---

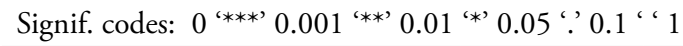

\begin{tabular}{l|c|c|c|c}
\hline Estrato & diff & lwr & upr & p adj \\
\hline Activo-Graduado & -2.2322 & -4.1786 & -0.2858 & 0.0198 \\
\hline Retirado-Graduado & 0.2047 & -1.0536 & 1.4631 & 0.9227 \\
\hline Retirado-Activo & 2.4370 & 0.2851 & 4.5888 & 0.0218 \\
\hline
\end{tabular}


Graduados frente a los Activos. En promedio la opinión de los retirados está por encima de la de los activos y la opinión de los graduados es mayor que la de los activos

En lo que respecta a las categorías de respuestas con menor puntaje (f, g, h, i, j), para el caso del ítem f) He recibido ayuda gubernamental para mi negocio, el análisis de varianza arroja los siguientes resultados:

\section{Cambios y diferencias en los oficios desempeñados}

En la Tabla 2, adjunta, se establecen las diferencias encontradas en torno a los tipos de trabajo u oficios desempeñados por los encuestados en el momento de la encuesta, o sea, después de su ingreso al Programa de Educación y Alfabetización.

Como se puede constatar en la Tabla 2, adjunta, el tipo de trabajo que realizan los estudiantes ACTIVOS está fuertemente asociado al Sector público (EsP), los RETIRADOS están más inclinados a tener "Empleo por contrato ocasional" (EcO), los GRADUADOS se inclinan al "Trabajo propio" aunque también hay asociación (aunque menos fuerte) con Empleado de empresa Privada, $(\mathrm{EeP})$ y Empleo por contrato indefinido (EcI)

\section{Oficios desempeñados antes de ingresar al programa}

De acuerdo con los datos de la Tabla 3, adjunta, la situación laboral ANTES $\mathrm{DE}$, de los estudiantes ACTIVOS, está más asociada con los oficios del Hogar $(\mathrm{OH})$,

Tabla 2

Cambios y diferencias en los oficios de la población después de su acceso al programa.

\begin{tabular}{l|c|c|c|c}
\hline Estrato & diff & lwr & upr & p adj \\
\hline Activo-Graduado & 1.1095 & 0.9693 & 1.270 & 0.1683 \\
\hline Retirado-Graduado & 1.0735 & 0.9814 & 1.174 & 0.1524 \\
\hline Retirado-Activo & 0.9675 & 0.8323 & 1.125 & 0.8638 \\
\hline
\end{tabular}

Análisis de varianza

Analysis of Variance Table 02

Response: $\log$ (datos [, variable[i]])

Df Sum Sq Mean Sq F value Pr $(>F)$

Estado 210.5122 .910 .055$.

Residuals 7411300.176

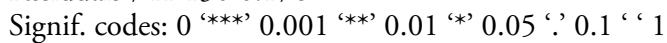

Number of cases in table: 727

Number of factors: 2

Test for independence of all factors:

Chisq $=59, \mathrm{df}=15, \mathrm{p}$-value $=3 \mathrm{e}-07$

Chi-squared approximation may be incorrect 
los GRADUADOS, con los oficios del Hogar, otros oficios, comerciantes y/o prestador de servicios y Vendedor en la calle. Los RETIRADOS, entre tanto, se inclinan más por otro tipo de oficios, oficios del Hogar y comerciantes o prestador de servicios

\section{Variación en el monto de los ingresos, por estratos}

Se procede a analizar el ingreso de la muestra comparado con los estratos, arrojando los resultados que se muestran a continuación:

Para analizar estos datos se ajusta un modelo log--lineal, con el nivel de ingreso como variable explicada y el estrato como variable explicativa. El resultado del ajuste es:

\section{Oportunidades de mejorar los ingresos, por categorías de estudiantes}

Con base en los datos observados se puede decir que las oportunidades (riesgo relativo) que tienen los estudiantes activos son 0,59 veces (menor que 1 , disminuye) de tener menos de un salario (<1 SM) a tener entre uno y dos salarios mínimos (1 a $2 \mathrm{SM}$ ) que los estudiantes retirados. En otras palabras: el riesgo relativo de pasar de un ingreso de menos de un salario a un ingreso de entre uno y dos salarios mínimos en los estudiantes activos es $3 / 5$ veces $(3 / 5=0,6$ aprox 0,59$)$ respecto a los retirados. Los activos son más propensos a tener menos de un salario mínimo que los retirados (Tablas 4 y 5 ).

De las Tablas 4 y 5 es claro la fuerte asociación entre los retirados y la categoría "uno a dos salarios mínimos" ( 1 a $2 \mathrm{SM}$ ) opuestos a los graduados quienes están

\section{Tabla 3}

Distribución porcentual de los Tipos de ocupación u oficios ANTES de acceder al Programa, por estratos.

\begin{tabular}{|c|c|c|c|c|c|c|c|c|}
\hline & Artesanías & $\begin{array}{c}\text { Comerciante } \\
\text { y/o prestador } \\
\text { de servicio }\end{array}$ & Música & $\begin{array}{c}\text { No } \\
\text { responde }\end{array}$ & $\begin{array}{c}\text { Oficios } \\
\text { del hogar }\end{array}$ & Otro & \begin{tabular}{|c|} 
Vendedor \\
de bienes \\
y servicios \\
en la calle
\end{tabular} & Sum \\
\hline Activo & 0.00 & 0.26 & 0.00 & 0.13 & 3.44 & 3.18 & 0.79 & 7.81 \\
\hline Graduado & 1.85 & 7.15 & 0.13 & 1.06 & 29.80 & 26.23 & 4.77 & 70.99 \\
\hline Retirado & 0.26 & 2.38 & 0.13 & 0.13 & 6.75 & 10.20 & 1.06 & 20.93 \\
\hline Faltante & 0.00 & 0.00 & 0.00 & 0.00 & 0.13 & 0.13 & 0.00 & 0.26 \\
\hline Sum & 2.12 & 9.80 & 0.26 & 1.32 & 40.13 & 39.74 & 6.62 & 100.00 \\
\hline $\begin{array}{l}\text { Prueba de } \\
\text { Number of } \\
\text { Number of } \\
\text { Test for in } \\
\text { Chisq = } \\
\text { Chi-squ }\end{array}$ & $\begin{array}{l}\text { ndependenc } \\
\text { cases in tab } \\
\text { factors: } 2 \\
\text { lependence } \\
16, \mathrm{df}=15 \text {, } \\
\text { ared approxi }\end{array}$ & $\begin{array}{l}\text { cia de Pearson } \\
\text { le: } 745 \\
\text { of all factors: } \\
\text { p-value }=0.4 \\
\text { imation may b }\end{array}$ & e incorr & & & & & \\
\hline
\end{tabular}


Tabla 4

Probabilidad de mejorar los ingresos por estratos.

\begin{tabular}{l|c|c|c}
\hline \multirow{2}{*}{ Referent group: } & $<\mathbf{1 ~ S M}$ & $\mathbf{1 ~ a ~ 2 ~ S M ~}$ & $>$ 2 SM \\
\cline { 2 - 4 } & Coeff. /SE & RRR $(\mathbf{9 5 \% C I})$ & Coeff./SE \\
\hline Intercept) & $\left(-0.36 / 0.163^{*}\right.$ & $-3.83 / 0.717^{* * *}$ & \\
\hline Estrato Activo & $-0.53 / 0.339$ & $0.59(0.3,1.15)$ & $1.56 / 0.888$ \\
\hline Estrato Graduado & $-0.89 / 0.194$ & $0.41(0.28,0.6)$ & $-0.11 / 0.801$ \\
\hline
\end{tabular}

Tabla 5

Probabilidad de mejorar los ingresos (Intercept).

\begin{tabular}{l|c}
\hline Estrato Activo & $4.76(0.84,27.14)$ \\
\hline Estrato Activo - Estrato Graduado & $0.9(0.19,4.32)$ \\
\hline
\end{tabular}

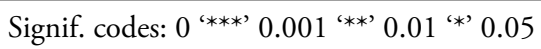

Residual Deviance: 969.93

$\mathrm{AIC}=981$

más asociados con la categoría "menos de un salario mínimo" "“< SM "). Los activos están más inclinados a la categoría "más de dos salarios mínimos" (">2 SM ") aunque la asociación no es tan fuerte como en los otros casos. En realidad los activos están "repartidos" entre ("<1 SM ") y (">2 SM ") porque según el resultado del análisis de correspondencias son las categorías más cercanas.

\section{Discusión}

El acceso a la educación que tuvo esta población si bien contribuyó a mejorar relativamente sus condiciones de vida en cuanto a tener mejores oportunidades para cambiar sus oficios y hacer un mejor aprovechamiento de sus recursos, ello por sí solo no les permitió una mejora en el nivel de sus ingresos, debido a que no contaron con los medios y las ayudas necesarias que les permitiera poner en práctica los conocimientos adquiridos en función de sus propios saberes y oficios, tal como se demuestra con las bajas calificaciones que dieron a esas alternativas de respuesta, demostrándose así una de las falencias de esta política educativa en cuanto a la carencia de apoyo institucional y acompañamiento en tal sentido.

La situación encontrada demuestra que efectivamente la educación es un medio eficaz de potenciación de las capacidades humanas que proporciona a las personas los conocimientos básicos para interpretar la realidad y las habilita para mejorar parcialmente su condición social y laboral, sin embargo los cambios identificados al respecto no reúnen las condiciones de un trabajo digno. En este sentido se confirma 
una de las tesis de Sen (2000) cuando dice que las perspectivas de empleo, salario y cambios en la vida de las personas van ligadas a las oportunidades que las instituciones les ofrecen y cuyo impacto será mayor si se interrelacionan y actúan complementariamente; los resultados obtenidos evidencian la desarticulación de esta política educativa con otras políticas socioeconómicas dirigidas a combatir la pobreza, desigualdad y exclusión, que respondan de manera integral a las necesidades y potencialidades de esta población acorde a sus características personales y del contexto, pues como bien se anotó anteriormente la mayoría de las personas beneficiarias de este programa de educación tanto en Córdoba como en Cartagena pertenecen al género femenino, son víctimas de la violencia y viven en condición de desplazamiento. Un alto porcentaje de estas mujeres son cabeza de familia, otra parte son indígenas y afrocolombianos, cuyo sustento depende del trabajo propio y de diversos oficios domésticos.

En el caso de Cartagena, por ejemplo, según el informe de avance de los logros de los Objetivos de Desarrollo del Milenio, se indica que el 34,2\% de la población cartagenera se encuentra en situación de pobreza, un indicador de $\mathrm{Ne}$ cesidades Básicas Insatisfechas de 26\%. La tasa de informalidad correspondió a un 59,4\%, que la sitúa casi 15 puntos porcentuales por encima de la meta establecida para el año 2015, de 45\% (PNUD; UNIVERSIDAD TECNOLÓGICA DE BOLÍVAR; INTERCONEXIÓN ELÉCTRICA ISA, 2012). Otros estudios encontraron que el desempleo en esta ciudad está focalizado principalmente en el grupo femenino y de jóvenes; que la tasa de desempleo de los hombres es casi tres veces inferior al de las mujeres. Se establece que la mayor generación de empleo en la ciudad se da por empleos de cuenta propia 57\% vs 53\%, en 2004 y 2011 (ALVIS ARRIETA; ESPINOSA ESPINOSA, 2011). Por su parte Carrillo Gullo y Valencia Jimenes (2010) concluyen que en esta zona del país el 77\% de la población joven y adulta permanecía por fuera del sistema escolar cuyas causas de deserción obedecían a la falta de oportunidades laborales.

La situación social del departamento de Córdoba, por su parte, no dista mucho de esta realidad. El Programa de la Naciones Unidas para el Desarrollo (PNUD, 2014) indica que en el 2012 la población de esta región del país presentaba un porcentaje de $60,2 \%$ en condiciones de pobreza, ubicándose en el tercer renglón a nivel nacional y en el primero de la costa caribe colombiana.

\section{Conclusiones}

Se concluye que la educación y alfabetización de jóvenes y adultos que se ofrece mediante diversas formas de atención y educación flexible no debe orientarse solamente en función de la empleabilidad y competitividad del mercado laboral, porque el acceso a dicho mercado no depende de la voluntad, el deseo ni el nivel educativo de las personas, sino de otros factores de carácter normativo, legal, e institucional impuesto por los grupos de poder y el Estado, como son, por ejemplo, 
ciertos requisitos, condiciones y trámites burocráticos establecidos por las empresas y las instituciones para la selección, evaluación y admisión de trabajadores, la mayoría de los cuales, como sucede casi siempre, no está al alcance de esta población, bien sea, porque no están debidamente capacitada o por razones de tradición, creencias y costumbres no están interesada en este modalidad de vinculación laboral; pero si desde los 'mundos del trabajo', en el entendido de la teoría marxista, es decir, como la condición básica y fundamental de toda la vida humana (ENGELS, 1981), como la expresión creadora y transformadora de toda actividad humana. $\mathrm{O}$, como lo sugiere Coraggio (2001), desde la economía del trabajo. Desde esta última perspectiva, la educación y alfabetización de adultos debería basarse en lo que las personas saben hacer y esperan perfeccionar para mejorar sus condiciones de vida.

En consecuencia se recomienda que los núcleos y/o ejes de los contenidos programáticos de este nivel educativo se articulen con los oficios y/o actividades identificados en este estudio los cuales se pueden clasificar así: Cultura, artesanías, oficios domésticos, construcción de obras civiles y mantenimiento de calles, vías y edificios; educación ambiental; servicios de transporte, compra y venta de bienes y servicios, entre otros.

Finalmente, se comparte el criterio de Solórzano (2005) para quien la educación y alfabetización debe ir más allá de ser un simple instrumento de adquisición de competencias para la empleabilidad y la productividad del trabajo, de carácter individual y aislada al servicio de un determinado sector económico y social; ya que ha de concebirse como un proceso histórico, plural, heterogéneo y contradictorio asociado a múltiples factores socioeconómicos y culturales que determinan su adquisición y desarrollo con distintos fines y bajo diferentes circunstancias, modo, tiempo y lugar.

\section{Notas}

1 Como respuesta a los lineamientos internacionales Colombia promulga el Decreto 3011 de 1997, reglamentario de la Ley 115 de 1994, por el cual se establecen normas para el ofrecimiento de la educación de adultos y se dictan otras disposiciones. El mencionado decreto establece los principios, los programas, las orientaciones curriculares especiales y las condiciones de organización y funcionamiento.

2 Esta modalidad educativa se compone de VI ciclos académicos así: ciclo I (1, 2 y 3 grados de la básica primaria); ciclo II (4 y 5 básica primaria); ciclo III (6 y 7 grados de la básica secundaria); ciclo IV (8 y 9 básica secundaria); ciclo V (grado 10 de la media vocacional) y ciclo VI (grado 11 de la media vocacional). La culminación de estos ciclos otorga el título de bachiller homologable a 11 años de la educación formal en Colombia.

3 Programa de Transferencia Monetaria Condicionada (TMC) que consiste en ofrecer apoyo económico a las familias colombianas en situación de vulnerabilidad. 


\section{Referencias}

ALVIS ARRIETA, J.; ESPINOSA ESPINOSA, A. Cartagena de Indias y los retos de la seguridad humana: Diagnóstico para una agenda de gobierno en la segunda década del siglo XXI. Economía y Región, Cartagena, v. 5, n. 2, p. 183-235, 2011.

CARRILLO GULLO, M.A.; VALENCIA JIMENEZ, N.N. Necesidades de aprendizaje para la vida. In: FUNDACIÓN TRANSFORMEMOS (Org.). Cartagena libre de analfabetismo: memorias de una hazaña. Bogotá: Fundación Transformemos, 2011.

CASAS, A.; CORTÉS, D.; GAMBOA, L.F. Desarrollo, bienestar y comparaciones interpersonales. Bogotá: Universidad del Rosario, 2001. n. 14. (Serie Documentos: Borradores de Investigación).

COMISIÓN ECONÓMICA PARA AMÉRICA LATINA Y EL CARIBE (CEPAL); OFICINA REGIONAL DE EDUCACIÓN DE LA UNESCO PARA AMÉRICA LATINA Y EL CARIBE (OREALC). Educación y conocimiento: eje de la transformación productiva con equidad. Santiago de Chile: CEPAL, 1992.

CONSEJO NACIONAL DE POLÍTICA ECONÓMICA E SOCIAL; REPUBLICA DE COLOMBIA; DEPARTAMENTO NACIONAL DE PLANEACIÓN. Documento CONPES 81. Consolidación del sistema nacional de formación para el trabajo en Colombia. Bogotá, 2004. 15p.

Documento CONPES 2947. Adecuación del SENA para la competitividad. Bogotá, 1997. 21p.

. Documento CONPES 3527. Política Nacional de Competitividad y Productividad. Bogotá, 2008. 87p.

Documento CONPES 3582. Política Nacional de Ciencia, Tecnología e Innovación. Bogotá, 2009. 69p.

CORAGGIO, J.L. Economía del trabajo: una alternativa racional a la incertidumbre. In: SEMINARIO INTERNACIONAL SOBRE ECONOMÍA Y ESPACIO. Conferencia presentada en el Panel Fronteras de la Teoría Urbana: CGE, Incertidumbre y Economía Popular, 2001, Belo Horizonte. 2001. Disponible en: <http://www.coraggioeconomia.org/jlc/ archivos\%20para\%20descargar/LaeconomadeltrabajoBH3.doc.pdf $>$. Acceso el: 01 ago. 2016.

DEPARTAMENTO NACIONAL DE PLANEACIÓN. Plan de Desarrollo 2010-2014: prosperidad para todos. Bogotá, 2010. 621p.

. Plan de Desarrollo 2014-2018: todos por un nuevo país. Paz, equidad y educación. Bogotá, 2014. 793p.

. Visión Colombia Segundo Centenario: 2019. Bogotá, 2009. 404p.

ENGELS, F. El papel del trabajo en la transformación del mono hombre. Moscú: Progreso, 1981.

FORO MUNDIAL SOBRE LA EDUCACIÓN. Educación para todos: cumplir nuestros compromisos comunes. Francia: UNESCO, 2000.

KALMAN, J. La importancia del contexto en la alfabetización. Revista Interamericana de Educación de Adultos, v. 24, n. 3, p. 11-27, 2002. 
MINISTERIO DE EDUCACIÓN NACIONAL. Plan Sectorial 2010-2014. Bogotá, 2010. 114p. n. 9.

ORGANIZACIÓN DE LAS NACIONES UNIDAS PARA LA EDUCACIÓN, LA CIENCIA Y LA CULTURA (UNESCO). Informe de seguimiento de la Educación en el Mundo 2016. La educación al servicio de los pueblos y el planeta: creación de futuros sostenibles para todos. París, 2016. Disponible en: <http://unesdoc.unesco.org/ images/0024/002457/245745s.pdf $>$. Acceso en: 25 oct. 2016.

Informe de seguimiento de la EPT en el mundo. La Educación para todos, 2000-2015: logros y desafíos. París, 2015. Disponible en: <http://unesdoc.unesco.org/ images/0023/002324/232435s.pdf>. Acceso en: 15 jul. 2016.

PROGRAMA DE LAS NACIONES UNIDAS PARA EL DESARROLLO (PNUD). Objetivo de desarrollo del Milenio Colombia 2014. Colombia: PNUD, 2014.

PROGRAMA DE LAS NACIONES UNIDAS PARA EL DESARROLLO (PNUD); UNIVERSIDADTECNOLÓGICA DE BOLÍVAR; INTERCONEXIÓN ELÉCTRICA ISA. Bolivar y Cartagena 2012: estado de avances de los objetivos de desarrollo del milenio. Bogotá: PNUD, 2012.

QUINTA CONFERENCIA INTERNACIONAL DE EDUCACIÓN DE LAS PERSONAS ADULTAS. La educación de las personas adultas. La declaración de Hamburgo. La agenda para el futuro. Hamburgo: UNESCO, 1997.

SEN, A. Desarrollo y libertad. Bogotá: Planeta, 2000.

SEXTA CONFERENCIA INTERNACIONAL DE EDUCACIÓN DE ADULTOS. Vivir y aprender para un futuro viable: el poder del aprendizaje de adultos. Brasil: UNESCO, 2010.

SOLÓRZANO, M.R. Modelo de evaluación del impacto social de losprogramas de alfabetización. Tesis (Doctoral) - Instituto Pedagógico Latinoamericano y Caribeño, Cuba, 2005.

TORRES, R.M. 12 Tesis para el cambio educativo. Justicia educativa y justicia económica. Informe de Fe y Alegría. España: Entreculturas, 2005.

Recibido el 13 de mayo de 2016.

Aprobado el 20 de febrero de 2017. 